\title{
Cetuximab in the treatment of head and neck cancer: preliminary results outside clinical trials
}

This article was published in the following Dove Press journal:

Cancer Management and Research

24 June 2010

Number of times this article has been viewed

\author{
Didier Dequanter \\ Mohammad Shahla \\ Pascal Paulus \\ Phillippe Lothaire \\ Department of Head and Neck \\ Surgery, CHU Charleroi, Montigny \\ le Tilleul, Belgium
}

Introduction: The purpose of this study was to evaluate the clinical efficacy in our daily practice, outside clinical trials, of cetuximab plus radiotherapy in a majority of treatment-naive patients with locoregionally advanced head and neck squamous cell carcinomas.

Methods: A retrospective study was performed to evaluate outcomes in patients who were treated definitively with cetuximab and radiotherapy (ExRT). Patients with stage III or IV, nonmetastatic, measurable squamous cell carcinoma of the head and neck (SCCHN) were eligible.

Results: There were 18 males and two females. The median age was 61 years (range from 49 to 87 years old). Concurrent radiotherapy and cetuximab was used, in first line, in 17 patients with locally advanced disease; two patients with recurrent SCCHN, who were intolerant of Cisplatin-based regimens, were treated with radiotherapy combined with weekly cetuximab; and 1 patient received cetuximab and radiotherapy postoperatively. The median time of response was 10 months (range from 2 to 24 months). A partial response was observed in 11 cases; a complete response in nine cases. The occurrence of grade 2-3 skin toxicity was observed in 11 cases. Skin toxicity was clearly correlated with a better response and the duration of the response to the treatment. The use of cetuximab in combination with radiotherapy does not increase the side effects of radiotherapy. At the end of the follow-up, 17 patients died.

Conclusion: Cetuximab, with its highly targeted mechanism of action and synergistic activity with current treatment modalities, is a valuable treatment option in head and neck patients. The effect of the epidermal growth factor receptor antagonist occurs without any change in the pattern and the severity of toxicity usually associated with head and neck radiation. Cetuximab seems not to provide the most benefit for patients with oropharyngeal cancers but will in patients with T4 tumors. However, the median duration of local control was less as described in the clinical trials.

Keywords: head and neck cancer, epidermal growth factor receptor antagonist, radiotherapy, clinical trials

\section{Introduction}

As $90 \%$ of all cases of squamous cell cancer of the head and neck show expression of epidermal growth factor receptor (EGFR), there is a strong rationale supporting EGFR targeting in this malignancy. This rationale is further strengthened by the fact that EGFR overexpression is associated with worse prognosis ${ }^{1,2}$ molecule consists of an $\mathrm{N}$ terminal, extracellular, ligand-binding domain; a hydrophobic transmembrane domain; and a C-terminal intracellular domain with tyrosine kinase activity. The EGFR can be stimulated by several growth factors such as transforming growth factor- $\alpha$ (TGF- $\alpha$ ) and 
EGF, which bind to the extracellular domain of the receptor. This ligand binding results in EGFR dimerization and activation of the tyrosine kinase domains present on each receptor. This kinase activation results in cross-phosporylation of tyrosine residues on each member of the receptor repair and initiates the formation of signalling complexes in the cytoplasm, which can translocate to the cell nucleus and activate gene transcription. This editing of gene transcription eventually result in the induction of several cellular responses such as cell proliferation. Ultimately receptor ligand complexes are internalized and the signal is terminated. Nowadays there are two EGFR targeting strategies used in clinical practice: monoclonal antibodies directed at the extracellular domain of the receptor and small molecule, and ATP tyrosine kinase inhibitors. ${ }^{4,5}$ The use of both modalities in the treatment of SCCHN is currently under investigation. Thus far, the monoclonal EGFR antibody cetuximab has been studied in detail. Cetuximab is a human-murine chimeric immunoglobulin $\mathrm{G}_{1}$ monoclonal antibody that competitively binds to the extracellular domain of the EGFR preventing activation of the receptor by endogenous ligands. The antibody-receptor complex is internalized and degraded resulting in a down regulation of the EGFR expression.

Cetuximab has been tested in metastatic/and or recurrent SCCHN both in first-line treatment in combination with platinum-based chemotherapy and as second-line after failure of platinum-based chemotherapy. Cetuximab has also been tested in association with radiation as definitive treatment for locoregionally advanced SCCHN.

The purpose of this study was to evaluate the clinical efficacy in our daily practice, outside clinical trials, of cetuximab plus radiotherapy in a majority of treatment-naive patients with locoregionally advanced head and neck squamous cell carcinomas.

\section{Methods}

A retrospective study was performed to evaluate outcomes in patients who were treated definitively with cetuximab and radiotherapy (ExRT). Only those patients judged to be medically suitable for definitive radiotherapy and who had a Karnofsky performance score of at least 60 with normal hematopoietic, hepatic, and renal function were eligible. Cetuximab and concurrent radiotherapy was used to treat 20 consecutive patients with squamous cell carcinoma of the head and neck (SCCHN), all of whom had tumors of the oral cavity, oropharynx, hypopharynx, or larynx. Patients with stage III or IV, nonmetastatic, measurable SCCHN were eligible. The prognostic significance of human papillomavirus
(HPV) status has only become clear in recent studies, and therefore no HPV testing was done systematically. Intravenous administration of cetuximab was initiated one week before radiotherapy at a loading dose of $400 \mathrm{mg} / \mathrm{m}^{2}$ followed by weekly administration of $250 \mathrm{mg} / \mathrm{m}^{2}$ for the duration of conventionally fractionated radiotherapy ( 70 Gy in 35 fractions of $2 \mathrm{~Gy}, 5$ fractions/week for 7 weeks). Tumor responses were assessed by computed tomography (CT) or magnet resonance imaging (MRI) at baseline and at 6-week intervals after the start of treatment until disease progression.

\section{Results}

There were 18 male and 2 female patients. The median age was 61 years (range from 49 to 87 -years-old). Nine patients presented an oropharyngeal tumor; seven patients had a hypopharyngeal tumor. The tumor was located at the oral cavity in two cases, and in the larynx in two cases. Seventeen patients were stage IV; three patients were stage III.

Concurrent radiotherapy and cetuximab was used as first line treatment in 17 patients with locally advanced disease; two patients with recurrent SCCHN and who were intolerant of cisplatin-based regimens were treated with radiotherapy combined with weekly cetuximab; and 1 patient received cetuximab and radiotherapy postoperatively. The median time of response was 10 months (range from 2 to 24 months). A partial response was observed in 11 cases; a complete response in nine cases. Among the nine patients with oropharyngeal tumor, the response was partial in six cases and complete in three cases. Among the patients with hypopharyngeal tumor, the response was partial in three cases and complete in four cases. After a follow-up of 33 months, the median survival time was 14 months.

The occurrence of grade 2-4 skin toxicity was observed in 11 cases. Skin toxicity was clearly correlated with a better response and the duration of the response to the treatment. The use of cetuximab in combination with radiotherapy does not increase the side effects of radiotherapy.

The cetuximab-induced rash began within 35 days of the initiation of treatment in $95 \%$ of patients. Of the patients who received cetuximab, 94\% received at least seven doses, and $90 \%$ received at least $1800 \mathrm{mg} / \mathrm{m}^{2}$. There was no association between cumulative dose and rash.

Patients with a prominent rash had longer survival compared with those with mild rash.

At the end of the follow-up, 17 patients died.

\section{Discussion}

It has been shown that cetuximab and radiation have a synergistic effect on human squamous cell carcinoma 
xenografts. ${ }^{1}$ Hence the rationale for combination radiotherapy with cetuximab in SCCHN is clearly present. ${ }^{2,3,4}$

In an initial study, cetuximab showed encouraging activity in patients with locally advanced SCCHN. In this phase I trial, ${ }^{5} 16$ patients with advanced SCCHN received treatment with cetuximab combined with either conventional or hyperfractionated radiotherapy. There was an impressive $100 \%$ response rate (13 complete and two partial responses) and interestingly, both treatments were generally well tolerated.

Furthermore, reported results showed better survival and locoregional disease control associated with cetuximab plus radiotherapy in patients with SCCHN, relative to radiotherapy alone, and these differences were not associated with reduced quality of life or increased radiation-induced mucositis or dysphagia. Indeed, in a multinational randomized study, ${ }^{6} 424$ patients with locoregionally advanced SCCHN were randomized to receive radiotherapy alone or radiotherapy combined with weekly cetuximab. Patients with stage III or IV, nonmetastatic, measurable squamous cell carcinoma of the oropharynx, hypopharynx or larynx were eligible. The primary endpoint was the duration of control of locoregional disease. Secondary endpoints were overall survival, progression free survival, the response rate, and safety. The median duration of locoregional control was 24.4 months with combined therapy and 14.9 months with radiotherapy alone $(P=0.005)$. The one-, two-, and threeyear rates of locoregional controls achieved with radiotherapy plus cetuximab $(63 \%, 50 \%, 47 \%)$ were significantly higher than those achieved with radiotherapy alone $(55 \%, 41 \%$, $34 \%$, respectively; $P<0.01$ for the comparison at three years). With a median follow up of 54 months the median survival time was 49 months among patients treated with combined therapy and 29.3 months among those given radiotherapy alone $(P=0.03)$.

In our study, the median time of response was 10 months (range from 2 to 24 months). A partial response was observed in 11 cases; a complete response in nine cases. After a follow-up of 33 months, the median survival time was 14 months.

An update analysis was done to assess additional 5-year overall survival results. ${ }^{7}$ The long-term results corroborate the earlier findings that the addition of cetuximab to radiotherapy improved the survival of patients with SCCHN. There was a difference of about $9 \%$ in absolute survival for the addition of cetuximab to radiotherapy compared with radiotherapy alone (36.4\% vs $45.6 \%$ ). Additionally, the subgroup analyses showed that patients given cetuximab who developed prominent cetuximab-induced acneiform rash (grade 2-4) had better overall survival compared with patients given cetuximab who developed a mild or not rash (grade 0-1). In our study, also, patients who developed grade $2-4$ rash had a better survival time than patients who developed no rash.

In their study, ${ }^{7}$ the overall survival benefit associated with the addition of cetuximab to radiotherapy relative to radiotherapy alone is remarkably similar to that seen in the 3-year analysis ( $45 \%$ vs $55 \%$ ), which supports the validity of 3 -year survival as a surrogate for long term overall survival.

The development of acneiform rash is a toxicity that is frequently associated with cetuximab and not radiotherapy alone. The characteristic cetuximab-induced acneiform rash rises during treatment and generally resolves completely in the first weeks following the cessation of the treatment. ${ }^{8}$ Of the 208 patients who received cetuximab and had information regarding skin toxicity, 174 (84\%) patients had rash and the incidence was similar to previous report of cetuximab alone. ${ }^{9} 127$ patients had prominent rash, the remaining 81 patients had mild rash or none. Patients with prominent rash had more than 2.5 times longer overall survival than did patients with mild rash.

Notably, cetuximab did not dramatically exacerbate the common toxic effects associated with radiotherapy of the head and neck, including mucositis, xerostomia, dysphagia, pain, weight loss, and performance status deterioration. ${ }^{10}$ However, a few cases of severe skin reactions within the radiation field were reported. ${ }^{11}$ In our study, each case of severe skin reaction was strongly correlated with a complete response to the treatment. As in other tumors the occurrence of grade 2-4 skin toxicity in SCHNN patients treated with cetuximab is associated with better outcome. ${ }^{12-14}$ In our study, skin toxicity was clearly correlated with a better response and the duration of the response to the treatment.

Furthermore, cetuximab seems to provide the most benefit for patients with oropharyngeal tumors, T1-3 tumors, treatment in the USA, concomitant boost, advanced nodal stage, and high Karnofosky Performance Scale. In our study, among the nine patients with an oropharyngeal tumor, a complete response was observed in only three cases, a partial response in six cases. Cetuximab seems to provide the most benefit for patient with $\mathrm{T} 4$ tumors. These subgroups represent small numbers of patients and therefore the results might represent spurious findings.

In conclusion, cetuximab, with its highly targeted mechanism of action and synergistic activity with current treatment modalities, is a valuable treatment option in head and neck 
patients. The effect of the EGFR antagonist occurs without any change in the pattern and the severity of toxicity usually associated with head and neck radiation. Cetuximab seems not to provide the most benefit for patients with oropharyngeal cancers but will in patients with T4 tumors. However, the median duration of local control was less as described in the clinical trials.

\section{Disclosure}

The authors report no conflicts of interest in this work.

\section{References}

1. Saleh MN, Raisch KP, Stackhouse MA, Grizzle WE, Bonner JA, Mayo MS. Combined modality therapy of A431 human epidermoid cancer using anti-EGFR antibody C225 and radiation. Cancer Biother Radiopharm. 1999;14:451-463.

2. Rapidis AD, Vermorken JB, Bourhis J. Targeted therapies in head and neck cancer: past, present and future. Rev Recent Clin Trials. 2008;3:156-166.

3. Borockstein B, Lacouture M, Agulnik M. The role of inhibitors of epidermal growth factor in management of head and neck cancer. J Natl Compr Canc Netw. 2008;7:696-706.

4. Langer CJ. Targeted therapy in head and neck cancer: state of the art 2007 and review of clinical applications. Cancer. 2008;12:2635-2645.

5. Robert F, Ezekiel MP, Spencer SA. Phase I study of anti-epidermal growth factor receptor antibody cetuximab in combination with radiation therapy in patients with advanced head and neck cancer. J Clin Oncol. 2001;19:3234-3243.

6. Bonner JA, Harrari P, Giralt J, Azarnia N, Shin DM, Cohen RB. Radiotherapy plus cetuximab for squamous cell carcinoma of the head and neck. N Eng J Med. 2006;354:567-578.
7. Bonner JA, Harari PM, Giralt J, Cohen B, Jones CH, Sur RJ, et al. Radiotherapy plus cetuximab for locoregionally advanced head and neck cancer: 5-year survival data from a phase 3 randomised trial, and relation between cetuximab-induced rash and survival. Lancet Oncol. 2010;11:21-28.

8. Bonner J, Harari PM, Giralt J. The relationship of cetuximab-induced rash and survival in patients with head and neck cancer treated with radiotherapy and cetuximab. Int J Radiat Oncol Biol Phys. 2005;63:S73.

9. Lenz JH, Cutsem EV, Khambata-Ford S. Multicenter phase II and translational study of cetuximab in metastatic colorectal carcinoma refratory to irinotecan, oxaliplatin, and fluoropyrimidines. J Clin Oncol. 2006;24:4914-4921.

10. Li B, Yuan M, Kim IA, Chang CM, Bernhard EJ, Shu HK. Mutant epidermal growth factor receptor displays increased signalling through the phospahtdylinosotol - 3 kibnase/AKT pathway and promotes radioresistance in cells of astrocytic origin. Oncogene. 2004;23:4594-4602.

11. Budach W, Bölke E, Horney B. Severe cutaneous reaction during radiation therapy with concurrent cetuximab. $N$ Eng $J$ Med. 2007;357:514-515.

12. Herbst RS, Arquette M, Shin DM, Dicke K, Vokes EE, Azarnia N. Phase II multicenter study of the epidermal growth factor antibody cetuximab and cisplatin for recurrent and refractory squamous cell carcinoma of the head and neck. J Clin Oncol. 2005;23:5568-5577.

13. Burtness B, Goldwasser M, Flood W, Mattar B, Forastiere AA; Eastern Cooperative Oncology Group. Phase III randomized trial of cisplatin plus placebo compared with cisplatin plus cetuximab in metastatic/ recurrent head and neck cancer: an Eastern Cooperative Oncology Group Study. J Clin Oncol. 2005;23:8646-8654.

14. Bernier J. Cetuximab in the treatment of head and neck cancer. Expert Rev Anticancer Ther. 2006;11:1539-1552.
Cancer Management and Research

\section{Publish your work in this journal}

Cancer Management and Research is an international, peer-reviewed open access journal focusing on cancer research and the optimal use of preventative and integrated treatment interventions to achieve improved outcomes, enhanced survival and quality of life for the cancer patient. The journal welcomes original research, clinical \& epidemiological

\section{Dovepress}

studies, reviews \& evaluations, guidelines, expert opinion \& commentary, case reports \& extended reports. The manuscript management system is completely online and includes a very quick and fair peerreview system, which is all easy to use. Visit http://www.dovepress.com/ testimonials.php to read real quotes from published authors. 